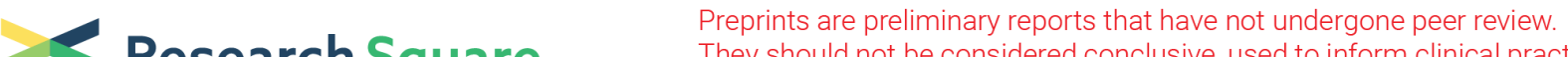 semem \\ A 1p/19q Codeletion Associated Immune Signature for Predicting Lower Grade Glioma Prognosis
}

Jie Xu

Huzhou Central Hospital, Affiliate Cent Hospital Huzhou University

Fang Liu

The Affiliated Changzhou No.2 Peoples' Hospital of Nanjing Medical University

Yuntao Li

Huzhou Cent Hospital, Affilated Huzhou Cent Hospital Huzhou University

Liang Shen ( $\nabla$ soochowneuro@163.com )

The affiliated changzhou NO.2 people's hospital of Nanjing Medical University https://orcid.org/0000$0002-8342-4153$

\section{Primary research}

Keywords: Lower grade gliomas (LGGs), immune, prognosis, Chinese Glioma Genome Atlas (CGGA), The Cancer Genome Atlas (TCGA)

Posted Date: June 15th, 2020

DOI: https://doi.org/10.21203/rs.3.rs-35118/v1

License: (c) (i) This work is licensed under a Creative Commons Attribution 4.0 International License. Read Full License

Version of Record: A version of this preprint was published at Cellular and Molecular Neurobiology on September 7th, 2020. See the published version at https://doi.org/10.1007/s10571-020-00959-3. 


\section{Abstract}

Background: Lower grade gliomas (LGGs) with codeletion of chromosomal arms 1p and 19q (1p/19 codeletion) has a favorable outcome. However, its overall survival (OS) varies. Here, we established an immune signature associated with 1p/19q codeletion for accurate prediction of prognosis of LGGs.

Methods: We extracted RNA sequencing and corresponding clinical data of LGGs from the Chinese Glioma Genome Atlas (CGGA) and The Cancer Genome Atlas (TCGA). The CGGA and TCGA databases were dichotomized into training group and testing group. The immune-related differentially expressed genes (DEGs) associated with 1p/19q codeletion were screened using Cox proportional hazards regression analyses. A prognostic signature was established using dataset from CGGA and tested in TCGA datasets. Subsequently, we explored the correlation between the prognostic signature and immune response.

Results: Thirteen immune genes associated with $1 \mathrm{p} / 19 \mathrm{q}$ codeletion were identified and used to construct a prognostic signature. The 1-, 3-, 5-year survival rates of the low-risk group were approximately $97 \%, 89 \%$, and $79 \%$, while those of the high-risk group were $81 \%, 50 \%$ and $34 \%$, respectively in the training group. The nomogram which comprised of age, world health organization (WHO) grade, primary or recurrent types, $1 p / 19 q$ codeletion status and risk score provided accurate prediction for the survival rate of glioma. DEGs that were highly expressed in the high-risk group clustered with many immune-related pathways. Immune checkpoints including T cell immunoglobulin domain and mucin domain 3 (TIM3), programmed cell death 1 (PD1), PD1 interacting with programmed death ligand 1 (PDL1), cytotoxic T lymphocyte antigen 4 (CTLA4), T cell immunoreceptor with Ig and ITIM domains (TIGIT), long non-coding RNA MIR 155 host gene (MIR155HG), and CD48 were correlated with the risk score. VAV3 and TNFRFSF11B were found to be candidate immune checkpoints.

Conclusion: The 1p/19q codeletion-associated immune signature provides accurate prediction of OS. VAV3 and TNFRFSF11B are novel immune checkpoints.

\section{Background}

Glioma, which derives from glial cells, is the commonest primary intracranial malignancy and is associated with poor outcomes. Gliomas are classified into grade I, II, III, or IV [1]. Those in histological grade IV, such as glioblastoma (GBM), are considered high-grade gliomas, while those in grade II and III are regarded as lower grade gliomas (LGG) [2]. The median GBM survival is 1 to 2 years after diagnosis while the overall survival (OS) for LGG patients ranges between 5 and 10 years. Despite advances in cancer screening, diagnosis and treatment, LGGs often progresses into high grade glioma within years [2-4]. While LGG patients experience a longer survival times and a better quality of life, progression into GBM, is associated with poor therapeutic options and significantly lower prognosis. Therefore, effective LGGs treatments are of utmost importance for improved glioma outcomes. 
Conventionally, brain tumors are classified through histogenesis, by observing microscopic tumor features. However, over time, it became clear that more efficient techniques were needed, leading to the development of molecular classification features techniques [5]. Currently, the WHO recommends that molecular parameters, such as the codeletion of chromosomal arms $1 p$ and $19 q(1 p / 19$ codeletion), and isocitrate dehydrogenase (IDH) mutation status be included in the histopathologic classification of brain cancers [6]. Numerous studies have associated 1p/19 co-deletions and IDH mutations with better glioma outcomes $[7,8]$. However, prognoses of glioma patients harboring $1 \mathrm{p} / 19$ codeletion vary widely [9]. Little is known about how $1 \mathrm{p} / 19$ codeletion affects LGG prognosis.

In glioma treatment, surgery, followed by chemotherapy and radiotherapy are associated with some improvement in therapeutic benefits relative to surgery alone. Immunotherapy is expected to improve treatment outcomes against glioma. While 1p/19 codeletion is being used in LGGs classification, little is known about the correlation between $1 \mathrm{p} / 19$ codeletion, the immune system and OS [10]. Here, we uncovered a prognostic immune signature that correlates with $1 \mathrm{p} / 19$ codeletion. We hypothesize that gene expression reprogramming that follows the $1 \mathrm{p} / 19$ codeletion might modulate the immune system.

\section{Material And Methods}

\section{Identification of immune-related genes correlated with 1p19q codeletion}

Glioma RNA-seq datasets and corresponding clinical information were downloaded CGGA (http://www.cgga.org.cn/) and TCGA (https://portal.gdc.cancer.gov/). Kaplan-Meier (KM) analysis was then used to evaluate survival. Log-rank tests were used to assess the correlation between $1 \mathrm{p} 19 \mathrm{q}$ codeletion status and OS in various WHO grade phenotypes. The 1p19q co-deletion status assessed using gene expression analysis as done previously $\mathrm{Hu}$ et al [9]. Next, univariate and multivariate Cox regression analyses were used to evaluate the value $1 \mathrm{p} 19 \mathrm{q}$ co-deletion as an independent prognostic factor. Differentially expressed genes (DEGs) in 1p19q co-deletion vs non-deleted samples were identified using the "limma" package in R software (version 3.6.1), by imposing the following criteria: |log2 foldchange, $\log 2 \mathrm{FC} \mid>1$ and an adjusted $p=<0.05$. This analysis involved data from $1921 \mathrm{p} 19 \mathrm{q}$ codeletion LGG samples and 394 non-deletion LGG samples. 1p19q co-deletion associated immune-related DEGs were identified from the DEGs based on immune-related gene annotation on the IMMPORT website (https://www.immport.org/) [11]. Only genes shared by CGGA and TCGA were included in downstream analyses. Only samples for which an OS time of $>90$ days were retained for downstream analyses.

\section{Elucidation of the prognostic signature}

Next, univariate Cox proportional hazards regression and LASSO (least absolute shrinkage and selection operator) Cox regression analyses were done on the 1p19q codeletion associated immune-related DEGs to prognosis-associated genes. The LASSO regression algorithm is used to reduce overfitting highdimensional prognostic genes $[12,13]$. Multivariate Cox proportional hazards regression analysis was then used to establish a prognostic signature with a coefficient $(\beta)$ based on all the genes included in the signature [14]. The risk score was a sum value calculated in accordance with the formulate: risk score= 
(expression of gene $A 1 * \beta 1)+($ expression of gene $A 2 * \beta 2)+($ expression of gene $A 3 * \beta 3)+\ldots$ (expression of gene $A n \star \beta n$ ) [15]. All CGGA dataset samples were identified and classified as either low risk or high-risk based on the median risk score [16]. KM survival plots and log-rank tests were used to evaluate the correlation between risk scores and OS.

\section{Validation of the prognostic signature}

Next, internal and external validation analyses were done to verify the prognostic signature's predictive power, which was evaluated using survival plots, 1, 3, and 5-year time-dependent receiver operating characteristic (ROC) curves, and survival status plots were [17]. Heatmaps and violin plots were used to visualize the expression profiles of the prognostic signature genes in the low and high-risk groups.

\section{Evaluation of the independent value of the prognostic signature}

Correlation between risk score and clinical information including age, sex, radiotherapy status, chemotherapy status, tumor grade, primary or recurrent tumor and IDH mutation status were analyzed. Univariate and multivariate Cox regression coupled with available clinical information were used to evaluate the independent prognostic capacity of the risk score.

\section{Prognostic nomogram analysis and validation}

Independent prognostic factors emerging from the CGGA dataset were subjected to nomogram analysis to predict the 1, 3 and 5-year survival [18]. 5 independent prognostic factors, age, WHO grade, primary or recurrent glioma types, $1 \mathrm{p} 19 \mathrm{q}$ codeletion status, and risk score, were used to develop the nomogram. We then validated the nomogram's prognosis accuracy using concordance index (C-index) combined with a calibration curve plot. This analysis was done in 1000 reiterations $[2,19]$.

\section{Gene ontology analysis}

Next, we executed a GO term analysis of DEGs between the high and low-risk groups. DEGs were identified by setting the following thresholds: $\log 2 \mathrm{FC}>1.2$ and $p$-value $<0.05$. Significantly enriched GO terms were indicated by $p$-value $<0.01, q$ value $<0.01$, and gene counts $>10$. Results from this analysis were visualized on circle plots.

\section{Analysis of correlation between risk score and expression of immune checkpoints}

Differential expression of 7 established immune checkpoint genes in the low and high-risk groups was analyzed. These are, T cell immunoglobulin domain and mucin domain 3 (TIM3), programmed cell death 1 (PD1), PD1 interacts with programmed death ligand 1 (PDL1), cytotoxic T lymphocyte antigen 4 (CTLA4), T cell immunoreceptor with Ig and ITIM domains (TIGIT), long non-coding RNA MIR 155 host gene (MIR155HG), and CD48 [20-24] . This analysis evaluated the correlation between risk score and expression of the checkpoint genes. P-value $=<0.05$ was considered statistically significant.

\section{Evaluation of the candidate immune checkpoints}


The following criteria used to elucidate underlying immune checkpoints: (1) DEGs in high-risk and lowrisk groups were common in the CGGA and TCGA datasets, (2) genes correlated with OS, (3) the genes were independent of clinical prognosis parameters including age, sex, WHO grade, and IDH1 mutation status, (4) genes had an AUC >0.7, (5) there was correlation between gene expression and risk score, (6) candidate immune checkpoints genes had a correlation value $>0.6$ both in CGGA and TCGA datasets, (7) candidate immune checkpoints show correlation with familiar immune checkpoints, (8) select candidates with a correlation score $>0.4$ as immune checkpoints.

\section{Results}

\section{$1 p / 19 q$ co-deletion and differentially expressed immune genes}

Analysis of survival in the CGGA datasets, revealed significantly lower OS in cases with $1 p / 19 q$ codeletion relative to those lacking the codeletion. Interestingly, outcomes were markedly in grade II and III tumors with 1p/19q co-deletion relative to grade IV tumors (Fig 1a). Univariate and multivariate Cox analyses revealed 1p/19q co-deletion as an independent prognostic factor for LGGs (Fig 1b-c). Differential gene expression analysis revealed that 551 DEGs between $1 p / 19 q$ co-deletion samples $(n=191)$ and non-codeletion samples $(n=393)$. Of the 551,56 are immune-related genes. No statistically significant differences were noted in co-deletion vs non-codeletion samples with regards to sex, age, primary or recurrent type, chemotherapy or radiotherapy status (Table 1).

\section{Elucidation and internal validation of the immune-related prognostic signature}

Univariate Cox proportional hazards regression and LASSO regression analyses of candidate genes revealed 23 genes that were screened in multivariate Cox proportional hazards regression. Thirteen immune-related genes associated with coefficients were included in the prognostic signature (Table 2). KM analysis of the low and high-risk LGG samples using log-rank test revealed that 1, 3 and 5-year survival rates in the low-risk group were $97 \%, 89 \%$, and $79 \%$, respectively, while in the high risk groups survival rates were $81 \%, 50 \%$ and $34 \%$, respectively (Fig $2 a$ ). The prognosis was significantly better in cases with lower risk scores, indicating that the risk score negatively correlated with OS. AUC values for the signature's prediction of 1,3 , and 5 -year survival were $0.818,0.793$, and 0.750 , respectively (Fig $2 \mathrm{~b}$ ). Indicating high risk score correlated with decreased survival (Fig 2c). The heatmap depicted the visual difference trends of transcript expression of genes incorporated in the signature between the high- and low-risk categories (Fig 2d). The violin plot presented a statistically differential expression between the two categories (Fig 2e).

\section{External validation of the prognostic signature}

The 1, 3 and 5-year survival in the TCGA database was $99 \%, 89 \%$, and $76 \%$ respectively, while in the testing cohort, the corresponding survival rates were only $84 \%, 51 \%$, and $36 \%$, respectively (Fig $3 a$ ). The capacity of the testing cohort to predict survival was very similar to that of the training cohort. ROC curve 
analysis was used to validate prediction accuracy. The AUC values for 1, 3 and 5-years survival were $0.896,0.785$ and 0.708 , respectively (Fig 3b). A similar trend was observed in the testing cohort (Fig 3c-e).

\section{Evaluation of the independent prognostic value}

Correlation between risk score and clinical parameters, including age, sex, radiotherapy and chemotherapy status, tumor grade, primary or recurrent types and IDH mutation status, was assessed. The value of risk score was lower in chemotherapy, $1 \mathrm{p} / 19$ codeletion, tumor grade II, primary and IDH mutation categories $(p<0.05$, Fig 4a). Univariate and multivariate Cox proportional hazards regression indicated that the risk score phenotype had independent prognostic value in the training testing cohort (Fig 4b-e).

\section{Nomogram analysis}

Nomogram analysis, using 5 prognostic markers (age, tumor grade, primary or recurrent type, risk score, and $1 p / 19$ codeletion status), was used to predict survival in the training set. Among these prognostic factors, risk score ranked a vital proportion in the total points (Fig 5a). To validate the accuracy of the individual assessment, concordance index (C-index) and calibration curve of the nomogram were evaluated for internal validation. The $\mathrm{C}$-index of the nomogram was 0.794 . The visualized calibration curve for probabilities for 1, 3 and 5-year OS revealed good agreement between the predicted nomogram and actual survival (Fig $5 \mathrm{~b}-\mathrm{d}$ ). Additionally, internal validation was done by randomly sampling $50 \%$ of the CGGA samples. The C-index was 0.797, and the calibration curves had goodness-off-fit (Fig 5e-g).

\section{Go term analysis}

503 genes were differentially expressed between low and high-risk groups. Of these, 255 had a log2FC $>1.2$, and 166 of them were included in the term GO analysis. The GO term analysis produced 33 terms that had gene counts $>10,12$ of which (including 37 DEGs) were associated with immune-related terms, including B cell receptor signaling pathway, lymphocyte-mediated immunity and humoral immune response (Fig 6).

\section{Elucidation of immune checkpoints and risk score}

Next, the relationship between 7 established immune checkpoints and risk score was evaluated (Fig 7). Expression of immune checkpoint genes, (except TIGIT), in the low-risk vs high-risk samples was statistically significant in the training and testing sets (Fig 7a-b). TIM3, MIR155, and CD48 exhibited the highest correlation (>0.4) in the training set (Fig 7c-i). In the testing set, TIM3, MIR155, PD1, and PDL1 expression positively correlated with risk score (Fig 7g-p). Analysis of the correlation between DEGs (in low vs high-risk samples) and survival indicated that 279 and 199 genes in the CGGA and TCGA dataset, respectively, are significantly associated with OS. Further analysis revealed 42 and 73 genes in the CGGA and TCGA datasets (AUC >0.7), respectively, that were independent of age, gender, tumor grade and IDH mutation status. Of these, 20 were common between the 2 datasets. Analysis of correlation between expression of the 20 genes and the risk score revealed 6 (colorectal neoplasia differentially expressed 
(CRNDE), transmembrane protein 71 (TMEM71), growth arrest specific 2 like 3 (GAS2L3), insulin like growth factor 2 mRNA binding protein 3 (IGF2BP3), vav guanine nucleotide exchange factor 3 (VAV3), TNF receptor superfamily member $11 \mathrm{~b}$ (TNFRSF11B)) with a correlation coefficient $>0.6$ in the training and he validation groups (Fig 8a-d). VAV3 and TNFRSF11B are immune-related genes. Sankey diagram analysis revealed co-expression between the 7 established immune checkpoint genes and the 6 immune checkpoint genes we identified. CD48, MIR155HG, PDL1 showed a strong relationship (Cor $>0.4, p<0.05)$ with other immune checkpoints in the training and testing set (Fig 8e-f). VAV3 exhibited a close relationship with MIR155HG, while TNFRSF11B correlated with MIR155HG and PD1 in the training and testing sets.

\section{Discussion}

$1 p / 19 q$ codeletion is a well-established biomarker [25], currently recommended by the WHO for tumor grade classification [26]. Here, we find that the $1 p / 19$ codeletion related immune genes have prognostic potential in LGG. To design an unbiased prognostic system, we uncovered a prognostic signature by analyzing LGGs RNA-seq and clinical data from CGGA and TCGA. This prognostic signature validated the hypothesis that improved outcomes upon $1 p / 19 q$ codeletion are associated with altered immunoregulation. In addition to the well-established immune checkpoints, including PD1 and TIM3, uncovered 6 novel immune checkpoint candidate. Numerous studies have previously described prognostic signatures for glioma [27-29]. Additionally, there has been a growing interest in glioma immunotherapy [30-33]. There is evidence that $1 p / 19 q$ codeletion correlates with significantly improved glioma prognosis. However, it remains unclear whether the codeletion's impact on outcomes are mediated via immune regulation. Here, we find that an immune-related prognostic signature associated with $1 p / 19 q$ codeletion might influence glioma prognosis. Unlike a previous study [34], our prognostic signature, based on a phenotype, decreased heterogeneity and increased prediction accuracy.

Immunotherapy has generated a lot of interest as a treatment for gliomas [35]. Deng et al [14] reported an IDH1 mutation prognostic signature and its association immune-related GO terms. Here, we find that highly expressed genes in high-risk group correlated with various immune-related pathways, including, B cell receptor signaling, lymphocyte-mediated immunity, and humoral immune response. Analysis of the microenvironment has shown that immune-related pathways influence behavior of glioma cells [36]. To evaluate the relationship between our prognostic signature and immunobiology, we evaluate its correlation with well-established immune checkpoint genes, and found that TIM3, PD1, PDL1, CTLA4, MIR155HG, and CD48, but not TIGIT [20, 21, 23], correlates with risk score. These findings are to some extent consistent with those by Deng et al [14]. Recent studies have highlighted the potential of immune checkpoints therapeutic targets. Here, we identified 6 novel immune checkpoint genes (VAV3, GAS2L3, IGF2BP3, CRNDE, TNFRSF11B, and TMEM71) that correlate with prognosis. VAV3 and TNFRSF11B had already been annotated as immune-related genes on IMMPORT (https://www.immport.org/). The expression of these genes also highly correlates with risk score and expression of 7 well-established immune checkpoints. Most of the candidate immune checkpoint genes have been previously associated with glioma [37-39]. Kiang et al [40] reported that CRNDE is elevated in glioma and might be modulated 
by EGFR signaling to promote gliomagenesis. However, there is little knowledge of the role of our candidate immune checkpoints in immune regulation of glioma. Further studies are needed to experimentally validate their involvement in glioma.

Although the phenotypes of glioma were classified according to molecular biomarkers [41], the OS of LGGs with $1 p / 19 q$ codeletion varies widely. It is clear that the $1 p / 19 q$ codeletion associated immune prognostic signature reduces variability, making prognosis more accurate. The immune response pathways associated with high-risk group raised several important questions, including, which immune checkpoint genes might regulate these immune response pathways. Glioma prognosis remains extremely poor, suggesting that the single immune therapy in use has failed to significantly improve OS. However, it should be noted that a diversified immune therapeutic strategy may be more effective. Thus, additional immune checkpoints for LGG treatment should be made first-line treatments along with surgical resection, radiotherapy or chemotherapy. The novel candidate immune checkpoint genes identified here, especially VAV3 and TNFRSF11B, are likely to become established immune checkpoint genes.

The purpose of this study was to establish a prognostic signature for LGGs with $1 p / 19 q$ co-deletion that can be used in clinical settings. However, LGGs including astrocytomas and oligodendrogliomas, have great tissue heterogeneity. The utility of this prognostic signature will likely be limited by such heterogeneity. The candidate immune checkpoint genes, are in fact, prognostic related genes and had a close correlation with the risk score and well-established immune checkpoints. The immune-related function and mechanisms of candidate immune checkpoints in LGGs in our study were hypothesized and their experimental validation is necessary. We contend that immunotherapy based on multiple immune checkpoints simultaneously may provide improved outcomes in glioma.

\section{Conclusions}

The $1 p / 19 q$ codeletion-associated immune signature provides accurate prediction of OS. VAV3 and TNFRFSF11B are novel immune checkpoints.

\section{Declarations}

Ethics approval and consent to participate

Not applicable. This article does not contain any studies with human participants performed by any of the authors.

Consent for publication

Not applicable.

Availability of data and materials 
The RNA-seq data and corresponding clinical information were observed from the TCGA (https://portal.gdc.cancer.gov/) and CGGA (http://www.cgga.org.cn). The immune-related gene list was got from the IMMPORT website (https://www.immport.org/).

\section{Competing interests}

The authors declare that they have no competing interests.

\section{Funding}

This work was supported by the Jiangsu Provincial Key Research and Development Program (No.BE2019652) and the Youth Program of Changzhou No.2 People's Hospital (No.2020K001).

Authors' contributions

All authors contributed to the study conception and design. Material preparation, data collection and analysis were performed by Jie Xu, Fang Liu and Liang Shen; The first draft of the manuscript was written by Jie Xu and Fang Liu and all authors commented on previous versions of the manuscript. All authors read and approved the final manuscript.

Acknowledgements

We would like to acknowledge the researchers' contribution to the TCGA, CGGA databases and support of the fund.

\section{Abbreviations}

Lower-grade gliomas (LGGs), The Cancer Genome Atlas (TCGA), Chinese Glioma Genome Atlas (CGGA), differentially expressed genes (DEGs), isocitrate dehydrogenase (IDH), receiver operating characteristic (ROC), least absolute shrinkage and selection operator (LASSO), RNA-Sequencing (RNA-Seq), log2Foldchange (log2FC), overall survival (OS), hazard ratios (HRs), confidence intervals (Cls), concordance index (C-index), coefficient (coef), world health organization (WHO), T cell immunoglobulin domain and mucin domain 3 (TIM3), programmed cell death 1 (PD1), PD1 interacting with programmed death ligand 1 (PDL1), cytotoxic T lymphocyte antigen 4 (CTLA4), T cell immunoreceptor with Ig and ITIM domains (TIGIT), long non-coding RNA MIR 155 host gene (MIR155HG), glioblastoma (GBM), KaplanMeier (KM), colorectal neoplasia differentially expressed (CRNDE), transmembrane protein 71 (TMEM71), growth arrest specific 2 like 3 (GAS2L3), insulin like growth factor 2 mRNA binding protein 3 (IGF2BP3), vav guanine nucleotide exchange factor 3 (VAV3), TNF receptor superfamily member $11 \mathrm{~b}$ (TNFRSF11B), adrenomedullin 2 (ADM2), coagulation factor II (thrombin) receptor-like 1 (F2RL1), neurotensin (NTS), cardiotrophin-like cytokine factor 1 (CLCF1), S100 calcium binding protein A3 (S100A3), bone morphogenetic protein 8b (BMP8B), family with sequence similarity 19 (chemokine (C-C motif)-like), member A3 (FAM19A3), T cell receptor delta constant (TRDC), prolactin releasing hormone receptor 
(PRLHR), androgen receptor (AR), glucagon-like peptide 1 receptor (GLP1R), gene ontology (GO), primary or recurrent (PR), radiotherapy (Radio), chemotherapy (Chemo), not available ( $\mathrm{Na})$

\section{References}

1. Louis DN, Ohgaki H, Wiestler OD, Cavenee WK, Burger PC, Jouvet A, et al. The 2007 WHO Classification of Tumours of the Central Nervous System. Acta Neuropathol. 2007;114:97-109. doi:10.1007/s00401-007-0243-4.

2. Kiran M, Chatrath A, Tang X, Keenan DM, Dutta A. A Prognostic Signature for Lower Grade Gliomas Based on Expression of Long Non-Coding RNAs. Mol Neurobiol. 2019;56:4786-98. doi:10.1007/s12035-018-1416-y.

3. Huang J, Samson P, Perkins SM, Ansstas G, Chheda MG, DeWees TA, et al. Impact of concurrent chemotherapy with radiation therapy for elderly patients with newly diagnosed glioblastoma: a review of the National Cancer Data Base. J Neurooncol. 2017;131:593-601. doi:10.1007/s11060016-2331-6.

4. Stupp R, Mason WP, van den Bent MJ, Weller M, Fisher B, Taphoorn MJB, et al. Radiotherapy plus concomitant and adjuvant temozolomide for glioblastoma. N Engl J Med. 2005;352:987-96. doi:10.1056/NEJMoa043330.

5. Louis DN. The next step in brain tumor classification: "Let us now praise famous men"... or molecules? Acta Neuropathol. 2012;124:761-2. doi:10.1007/s00401-012-1067-4.

6. Louis DN, Perry A, Reifenberger G, Deimling A von, Figarella-Branger D, Cavenee WK, et al. The 2016 World Health Organization Classification of Tumors of the Central Nervous System: a summary. Acta Neuropathol. 2016;131:803-20. doi:10.1007/s00401-016-1545-1.

7. Park YW, Han K, Ahn SS, Bae S, Choi YS, Chang JH, et al. Prediction of IDH1-Mutation and 1p/19qCodeletion Status Using Preoperative MR Imaging Phenotypes in Lower Grade Gliomas. AJNR Am J Neuroradiol. 2018;39:37-42. doi:10.3174/ajnr.A5421.

8. Leeper HE, Caron AA, Decker PA, Jenkins RB, Lachance DH, Giannini C. IDH mutation, 1p19q codeletion and ATRX loss in WHO grade II gliomas. Oncotarget. 2015;6:30295-305.

9. Hu X, Martinez-Ledesma E, Zheng S, Kim H, Barthel F, Jiang T, et al. Multigene signature for predicting prognosis of patients with $1 \mathrm{p} 19 \mathrm{q}$ co-deletion diffuse glioma. Neuro-oncology. 2017;19:786-95. doi:10.1093/neuonc/now285.

10. Ceccarelli M, Barthel FP, Malta TM, Sabedot TS, Salama SR, Murray BA, et al. Molecular Profiling Reveals Biologically Discrete Subsets and Pathways of Progression in Diffuse Glioma. Cell. 2016;164:550-63. doi:10.1016/j.cell.2015.12.028.

11. Zhang M, Zhu K, Pu H, Wang Z, Zhao H, Zhang J, Wang Y. An Immune-Related Signature Predicts Survival in Patients With Lung Adenocarcinoma. Front Oncol. 2019;9:1314. doi:10.3389/fonc.2019.01314. 
12. Castro DM, Veaux NR de, Miraldi ER, Bonneau R. Multi-study inference of regulatory networks for more accurate models of gene regulation. PLoS Comput Biol. 2019;15:e1006591. doi:10.1371/journal.pcbi.1006591.

13. Goeman JJ. L1 penalized estimation in the Cox proportional hazards model. Biom J. 2010;52:70-84. doi:10.1002/bimj.200900028.

14. Deng $X$, Lin D, Chen B, Zhang X, Xu X, Yang Z, et al. Development and Validation of an IDH1Associated Immune Prognostic Signature for Diffuse Lower-Grade Glioma. Front Oncol. 2019;9:1310. doi:10.3389/fonc.2019.01310.

15. Qian Z, Li Y, Fan X, Zhang C, Wang Y, Jiang T, Liu X. Prognostic value of a microRNA signature as a novel biomarker in patients with lower-grade gliomas. J Neurooncol. 2018;137:127-37. doi:10.1007/s11060-017-2704-5.

16. Liu B, Liu J, Liu K, Huang H, Li Y, Hu X, et al. A prognostic signature of five pseudogenes for predicting lower-grade gliomas. Biomed Pharmacother. 2019;117:109116. doi:10.1016/j.biopha.2019.109116.

17. Yang Z, Shang J, Li N, Zhang L, Tang T, Tian G, Chen X. Development and validation of a 10-gene prognostic signature for acute myeloid leukaemia. J Cell Mol Med 2020. doi:10.1111/jcmm.15109.

18. Long J, Wang A, Bai Y, Lin J, Yang X, Wang D, et al. Development and validation of a TP53associated immune prognostic model for hepatocellular carcinoma. EBioMedicine. 2019;42:363-74. doi:10.1016/j.ebiom.2019.03.022.

19. Duan J, Xie Y, Qu L, Wang L, Zhou S, Wang Y, et al. A nomogram-based immunoprofile predicts overall survival for previously untreated patients with esophageal squamous cell carcinoma after esophagectomy. J Immunother Cancer. 2018;6:100. doi:10.1186/s40425-018-0418-7.

20. Liu S, Wang Z, Wang Y, Fan X, Zhang C, Ma W, et al. PD-1 related transcriptome profile and clinical outcome in diffuse gliomas. Oncoimmunology. 2018;7:e1382792.

doi:10.1080/2162402X.2017.1382792.

21. Peng L, Chen Z, Chen Y, Wang X, Tang N. MIR155HG is a prognostic biomarker and associated with immune infiltration and immune checkpoint molecules expression in multiple cancers. Cancer Med. 2019;8:7161-73. doi:10.1002/cam4.2583.

22. Liu F, Huang J, Liu X, Cheng Q, Luo C, Liu Z. CTLA-4 correlates with immune and clinical characteristics of glioma. Cancer Cell Int. 2020;20:7. doi:10.1186/s12935-019-1085-6.

23. Hung AL, Maxwell R, Theodros D, Belcaid Z, Mathios D, Luksik AS, et al. TIGIT and PD-1 dual checkpoint blockade enhances antitumor immunity and survival in GBM. Oncoimmunology. 2018;7:e1466769. doi:10.1080/2162402X.2018.1466769.

24. Filippova N, Yang X, An Z, Nabors LB, Pereboeva L. Blocking PD1/PDL1 Interactions Together with MLN4924 Therapy is a Potential Strategy for Glioma Treatment. J Cancer Sci Ther. 2018;10:190-7. doi:10.4172/1948-5956.1000543.

25. Durand KS, Guillaudeau A, Weinbreck N, DeArmas R, Robert S, Chaunavel A, et al. 1p19q LOH patterns and expression of p53 and Olig2 in gliomas: relation with histological types and prognosis. 
Mod Pathol. 2010;23:619-28. doi:10.1038/modpathol.2009.185.

26. Ceccarelli M, Barthel FP, Malta TM, Sabedot TS, Salama SR, Murray BA, et al. Molecular Profiling Reveals Biologically Discrete Subsets and Pathways of Progression in Diffuse Glioma. Cell. 2016;164:550-63. doi:10.1016/j.cell.2015.12.028.

27. Jang B-S, Kim IA. A radiosensitivity gene signature and PD-L1 predict the clinical outcomes of patients with lower grade glioma in TCGA. Radiother Oncol. 2018;128:245-53. doi:10.1016/j.radonc.2018.05.003.

28. Zhang Y, Li J, Yi K, Feng J, Cong Z, Wang Z, et al. Elevated signature of a gene module coexpressed with CDC20 marks genomic instability in glioma. Proc Natl Acad Sci U S A. 2019;116:6975-84. doi:10.1073/pnas.1814060116.

29. Zhou Z, Huang R, Chai R, Zhou X, Hu Z, Wang W, et al. Identification of an energy metabolism-related signature associated with clinical prognosis in diffuse glioma. Aging (Albany NY). 2018;10:3185209. doi:10.18632/aging.101625.

30. Chheda ZS, Kohanbash G, Okada K, Jahan N, Sidney J, Pecoraro M, et al. Novel and shared neoantigen derived from histone 3 variant H3.3K27M mutation for glioma T cell therapy. J Exp Med. 2018;215:141-57. doi:10.1084/jem.20171046.

31. Weller M, Roth P, Preusser M, Wick W, Reardon DA, Platten M, Sampson JH. Vaccine-based immunotherapeutic approaches to gliomas and beyond. Nat Rev Neurol. 2017;13:363-74. doi:10.1038/nrneurol.2017.64.

32. Kohanbash G, Carrera DA, Shrivastav S, Ahn BJ, Jahan N, Mazor T, et al. Isocitrate dehydrogenase mutations suppress STAT1 and CD8+ T cell accumulation in gliomas. J Clin Invest. 2017;127:142537. doi:10.1172/JCl90644.

33. Deumelandt K, Platten M, Ochs K. Synergy of vaccination and agonist OX40 treatment-toward a mechanism-driven combination of glioma immunotherapy. Neuro-oncology. 2018;20:4-5. doi:10.1093/neuonc/nox169.

34. Zhang G-H, Zhong Q-Y, Gou X-X, Fan E-X, Shuai Y, Wu M-N, Yue G-J. Seven genes for the prognostic prediction in patients with glioma. Clin Transl Oncol. 2019;21:1327-35. doi:10.1007/s12094-01902057-3.

35. Srinivasan VM, Ferguson SD, Lee S, Weathers S-P, Kerrigan BCP, Heimberger AB. Tumor Vaccines for Malignant Gliomas. Neurotherapeutics. 2017;14:345-57. doi:10.1007/s13311-017-0522-2.

36. He S-J, Gu Y-Y, Yu L, Luo B, Fan R, Lin W-Z, et al. High expression and frequently humoral immune response of melanoma-associated antigen D4 in glioma. Int J Clin Exp Pathol. 2014;7:2350-60.

37. Salhia B, Tran NL, Chan A, Wolf A, Nakada M, Rutka F, et al. The guanine nucleotide exchange factors trio, Ect2, and Vav3 mediate the invasive behavior of glioblastoma. Am J Pathol. 2008;173:1828-38. doi:10.2353/ajpath.2008.080043.

38. Pop S, Enciu A-M, Necula LG, Tanase C. Long non-coding RNAs in brain tumours: Focus on recent epigenetic findings in glioma. J Cell Mol Med. 2018;22:4597-610. doi:10.1111/jcmm.13781. 
39. Kryvdiuk IV, Minchenko DO, Hlushchak NA, Ratushna OO, Karbovskyi LL, Minchenko OH. INHIBITION OF IRE1 MODIFIES EFFECT OF GLUCOSE DEPRIVATION ON THE EXPRESSION OF TNFa-RELATED GENES IN U87 GLIOMA CELLS. Ukr Biochem J. 2015;87:36-51. doi:10.15407/ubj87.06.036.

40. Kiang KM-Y, Zhang X-Q, Zhang GP, Li N, Cheng SY, Poon M-W, et al. CRNDE Expression Positively Correlates with EGFR Activation and Modulates Glioma Cell Growth. Target Oncol. 2017;12:353-63. doi:10.1007/s11523-017-0488-3.

41. Reifenberger G, Wirsching H-G, Knobbe-Thomsen CB, Weller M. Advances in the molecular genetics of gliomas - implications for classification and therapy. Nat Rev Clin Oncol. 2017;14:434-52. doi:10.1038/nrclinonc.2016.204.

\section{Tables}

Table 1 The characteristics of samples in CGGA 


\begin{tabular}{|c|c|c|c|c|c|}
\hline \multirow[t]{2}{*}{ Variables } & \multicolumn{3}{|c|}{ 1p19q codeletion } & \multirow[t]{2}{*}{ Value } & \multirow[t]{2}{*}{$P$ value } \\
\hline & Yes (191) & & No (393) & & \\
\hline Sex & & 1.816 & 0.178 & & \\
\hline Female & 88 & & 158 & & \\
\hline Male & 103 & & 235 & & \\
\hline Age (mean $\pm S D$, years) & $40.7 \pm 8.6$ & & $39.6 \pm 10.8$ & -1.034 & 0.181 \\
\hline $\mathrm{OS}\left(\right.$ mean, years) $^{\dagger}$ & $9.1(174)$ & & $5.6(363)$ & 66.680 & $<0.001^{\star \star \star}$ \\
\hline PR type & 2.926 & 0.087 & & & \\
\hline Primary & 137 & & 254 & & \\
\hline Recurrent & 54 & & 139 & & \\
\hline Histology type & 66.833 & $<0.001^{\star \star \star}$ & & & \\
\hline Astrocytoma & 7 & & 89 & & \\
\hline Oligodendroglioma & 95 & & 81 & & \\
\hline Mixed glioma & 89 & & 223 & & \\
\hline WHO grade & -20.558 & $<0.001^{\star * \star}$ & & & \\
\hline WHO II & 102 & & 170 & & \\
\hline WHO III & 89 & & 223 & & \\
\hline Radio status & 0.412 & 0.814 & & & \\
\hline Yes & 146 & & 291 & & \\
\hline No & 31 & & 69 & & \\
\hline $\mathrm{Na}$ & 14 & & 33 & & \\
\hline Chemo status & 1.961 & 0.375 & & & \\
\hline Yes & 97 & & 223 & & \\
\hline No & 69 & & 128 & & \\
\hline $\mathrm{Na}$ & 25 & & 42 & & \\
\hline IHD mutation status & 57.670 & $<0.001^{* \star \star}$ & & & \\
\hline Yes & 166 & & 246 & & \\
\hline No & 7 & & 124 & & \\
\hline $\mathrm{Na}$ & 18 & & 23 & & \\
\hline
\end{tabular}


PR primary or recurrent, OS overall survival, Radio radiotherapy, Chemo chemotherapy, Na not available, † 174 codeletion and 363 no codeletion samples were included using log-rank test according to the missing data.

Table 2: Thirteen genes and coefficients in the prognostic signature

\begin{tabular}{llll} 
Gene & Coef & Gene & Coef \\
\hline S100A3 & 0.010009781 & F2RL1 & 0.061144932 \\
\hline FAM19A3 & 0.048040197 & VAV3 & 0.053997988 \\
\hline ADM2 & 0.002940087 & BMP8B & 0.14087201 \\
\hline CLCF1 & 0.021229638 & NTS & 0.021282164 \\
\hline TNFRSF11B & 0.013799876 & AR & 0.033614978 \\
\hline GLP1R & -0.000625507 & PRLHR & -0.084033137 \\
\hline TRDC & 0.039746687 & &
\end{tabular}

ADM2: adrenomedullin 2, F2RL1: coagulation factor II (thrombin) receptor-like 1, NTS: neurotensin, VAV3: vav 3 guanine nucleotide exchange factor, CLCF1: cardiotrophin-like cytokine factor 1, S100A3: S100 calcium binding protein A3, BMP8B: bone morphogenetic protein 8b, FAM19A3: family with sequence similarity 19 (chemokine (C-C motif)-like), member A3, TRDC: T cell receptor delta constant, PRLHR: prolactin releasing hormone receptor, AR: androgen receptor, TNFRSF11B: tumor necrosis factor receptor superfamily, member 11b, GLP1R: glucagon-like peptide 1 receptor, coef: coefficient

\section{Figures}


a
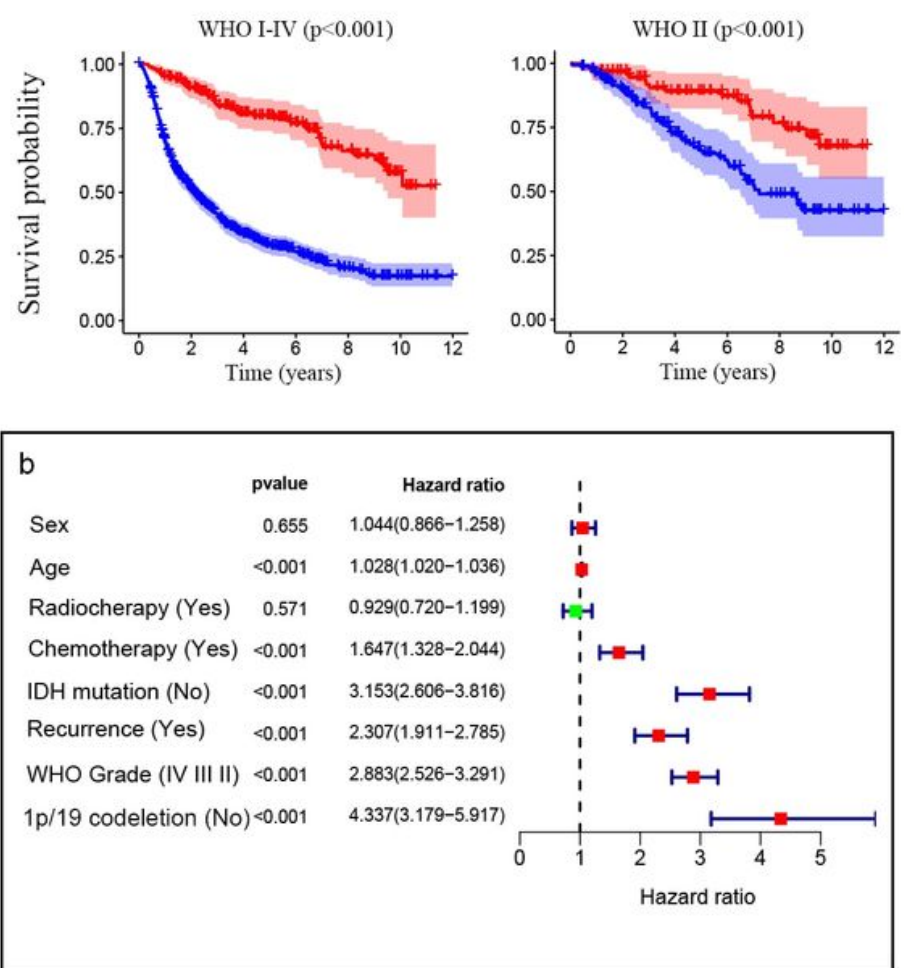

Non-codeletion
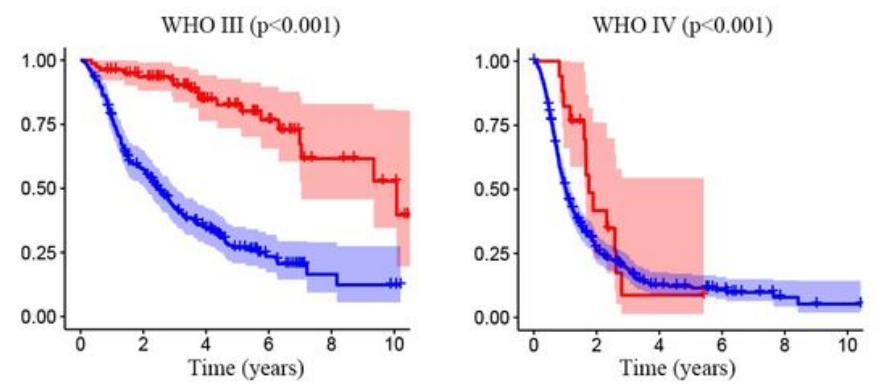

\section{Figure 1}

Kaplan-Meier (KM) survival curves illustrate that $1 p / 19 q$ codeletion predicts favorable outcomes in gliomas (a). 1p/19q codeletion is an independent factor for predicting OS in univariate (b) and multivariate (c) Cox analyses. 


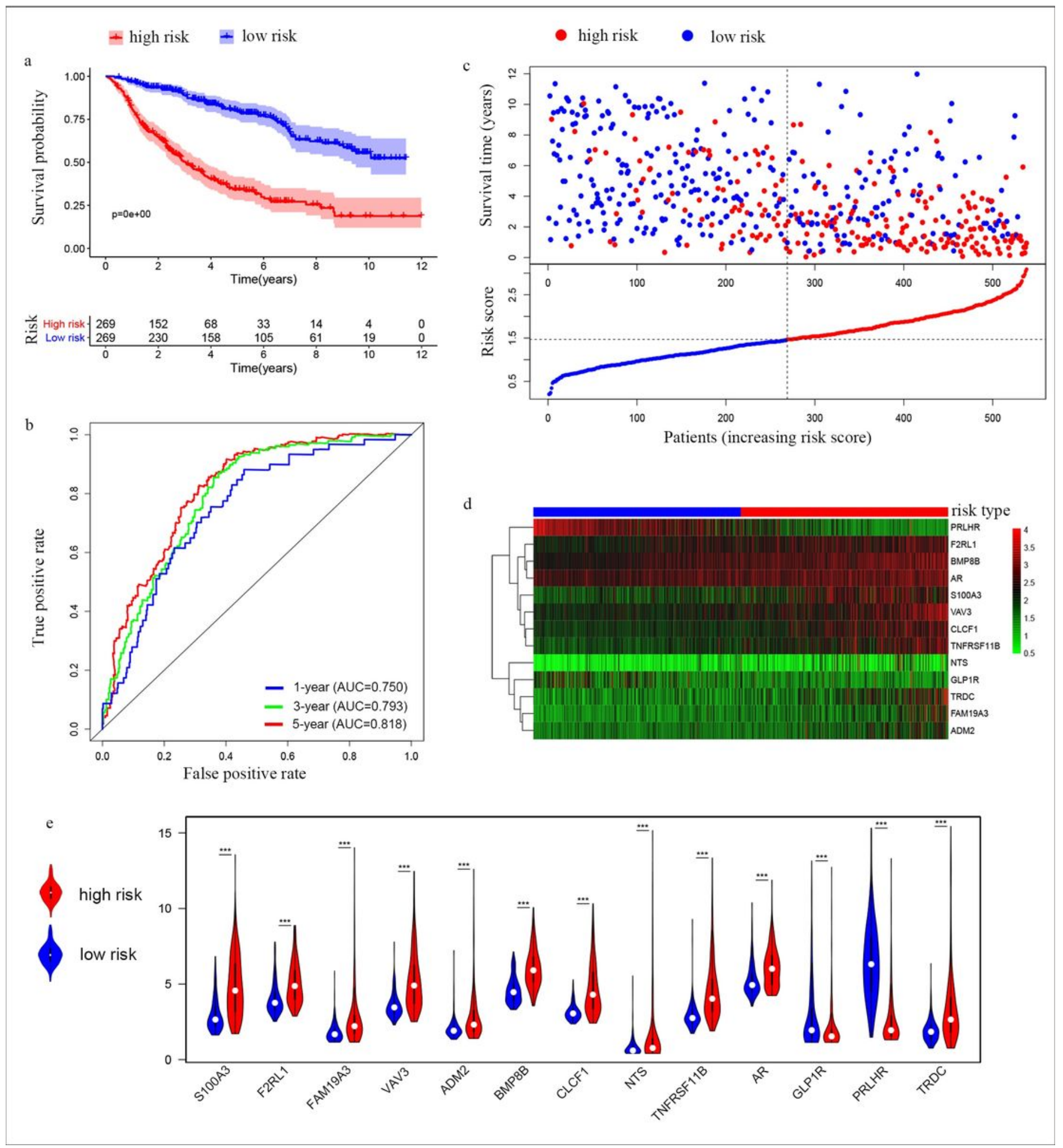

Figure 2

The prognostic signature validated in the training cohort. The KM survival curve of OS for LGGs (a); The time-dependent receiver operating characteristic (ROC) curves for the 1-, 3- and 5-year survival rate (b); The survival status of each samples and the distribution of risk scores (c); The heatmap (d) and violin plot (e) of 13 genes between the low- and high-risk groups included the signature. 


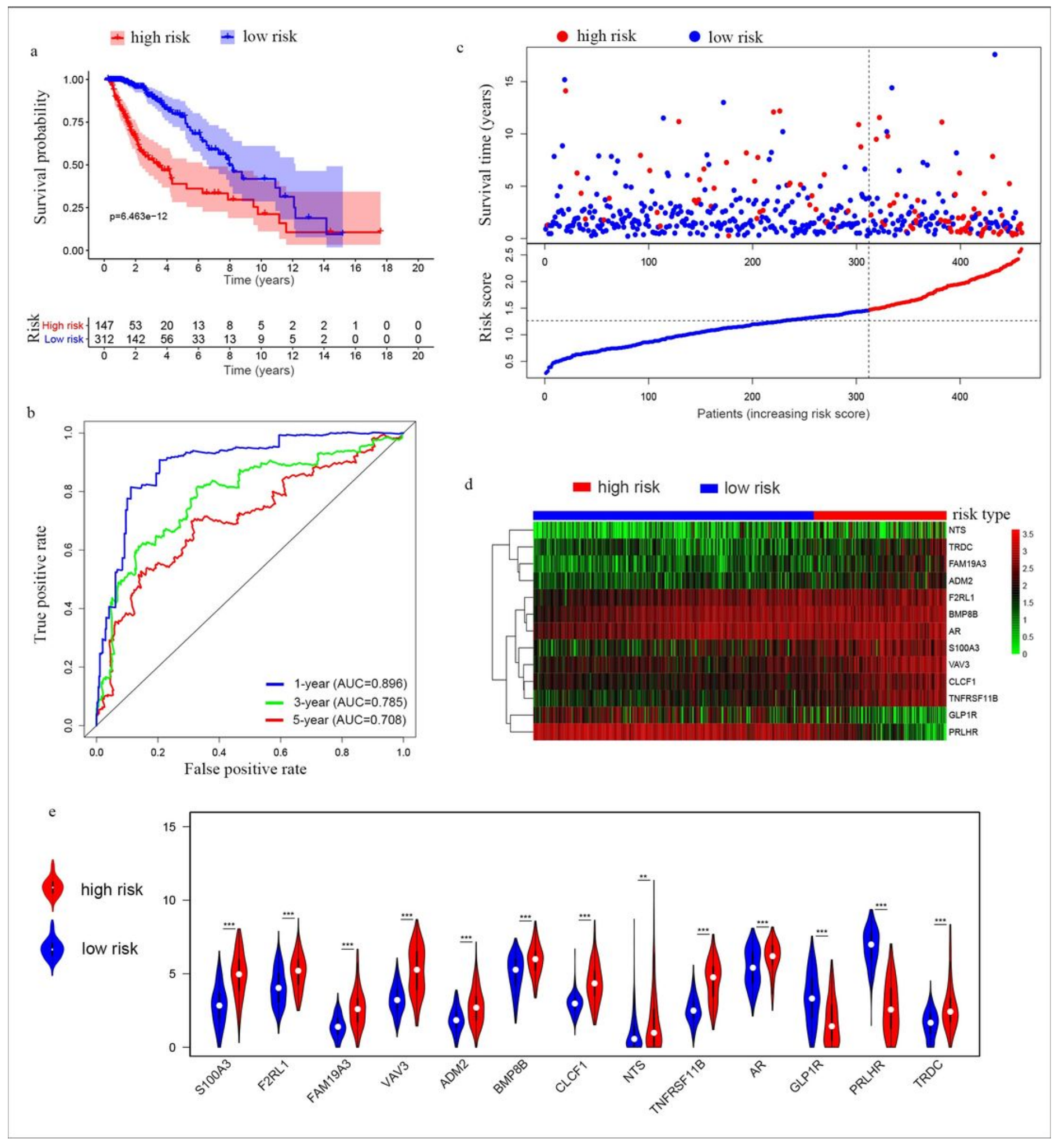

\section{Figure 3}

The prognostic signature validated in the testing cohort. The KM survival curve of OS for LGGs (a); The time-dependent ROC curves for 1-, 3- and 5-year survival rate (b); The survival status of each samples and the distribution of risk scores (c); The heatmap (d) and violin plot (e) of 13 genes between the low- and high-risk groups included the signature. 

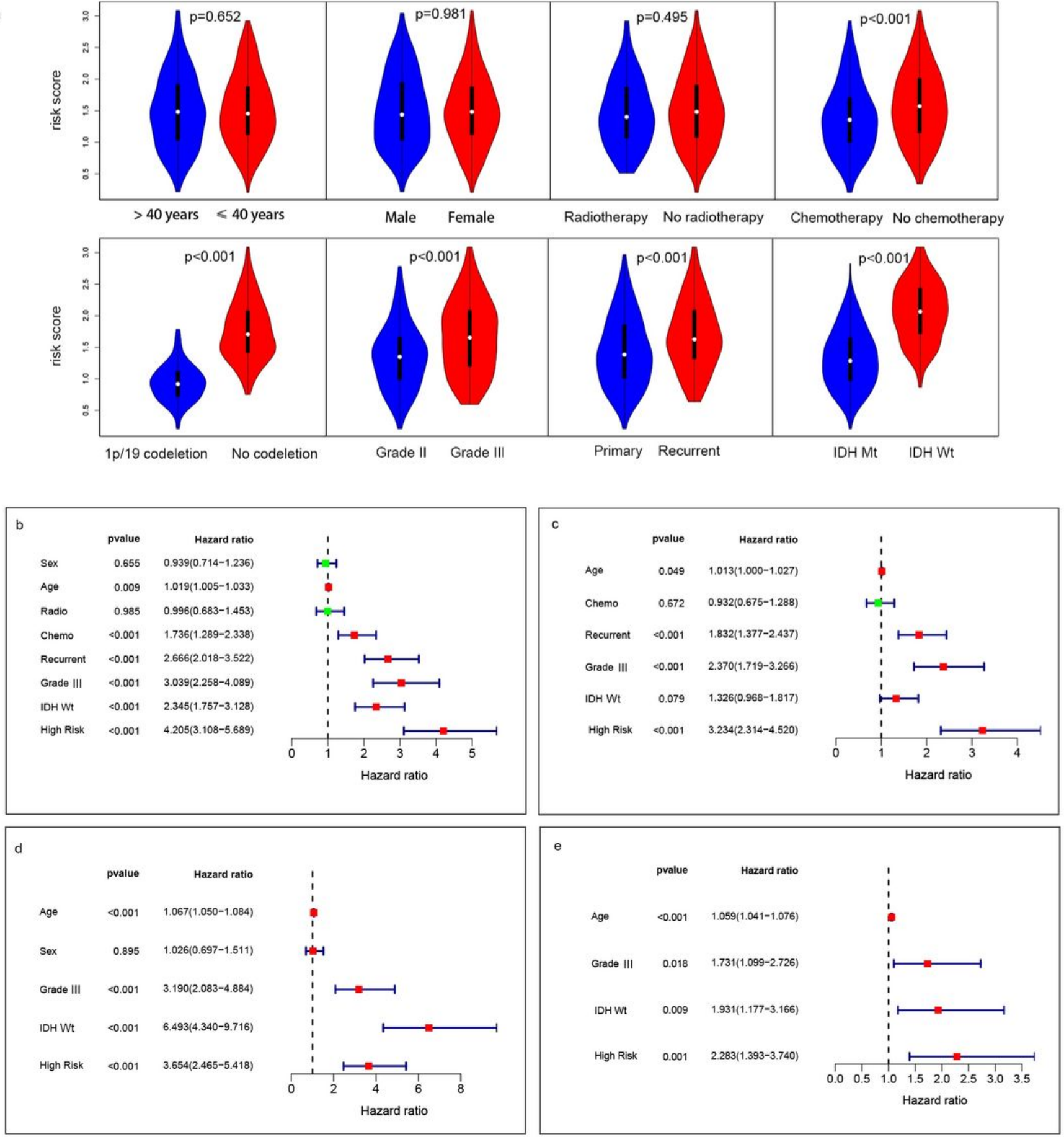

Figure 4

Lower risk scores in patients receiving chemotherapy, with 1p/19q codeletion, WHO grade II, primary tumor, or IDH mutation. However, risk score in age $>40$ years, male, or radiotherapy groups, has no statistical significance (a). univariate (b) and multivariate (c) Cox proportional hazards regression analyses showing the risk score is an independent predictor of the OS. 


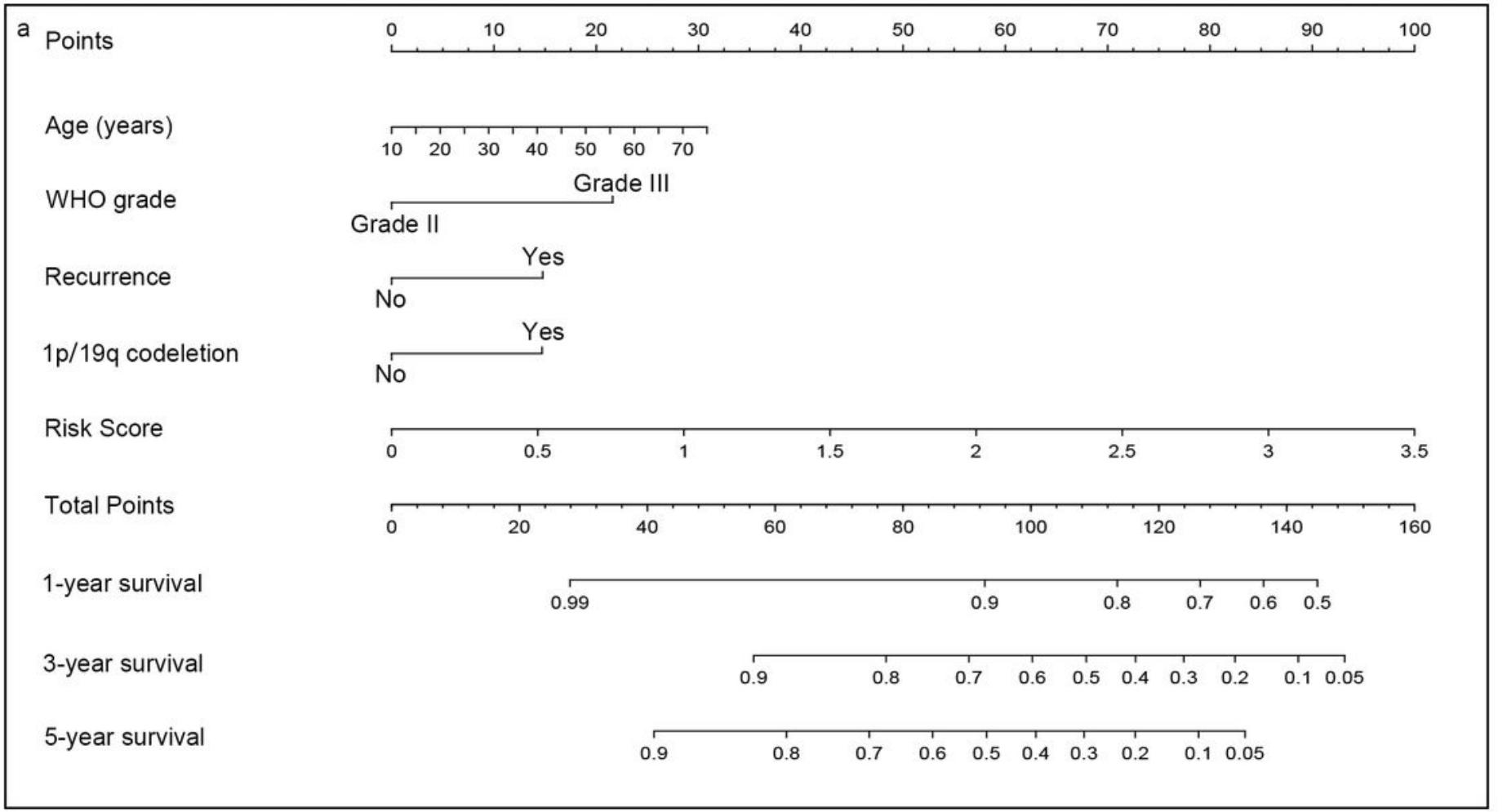

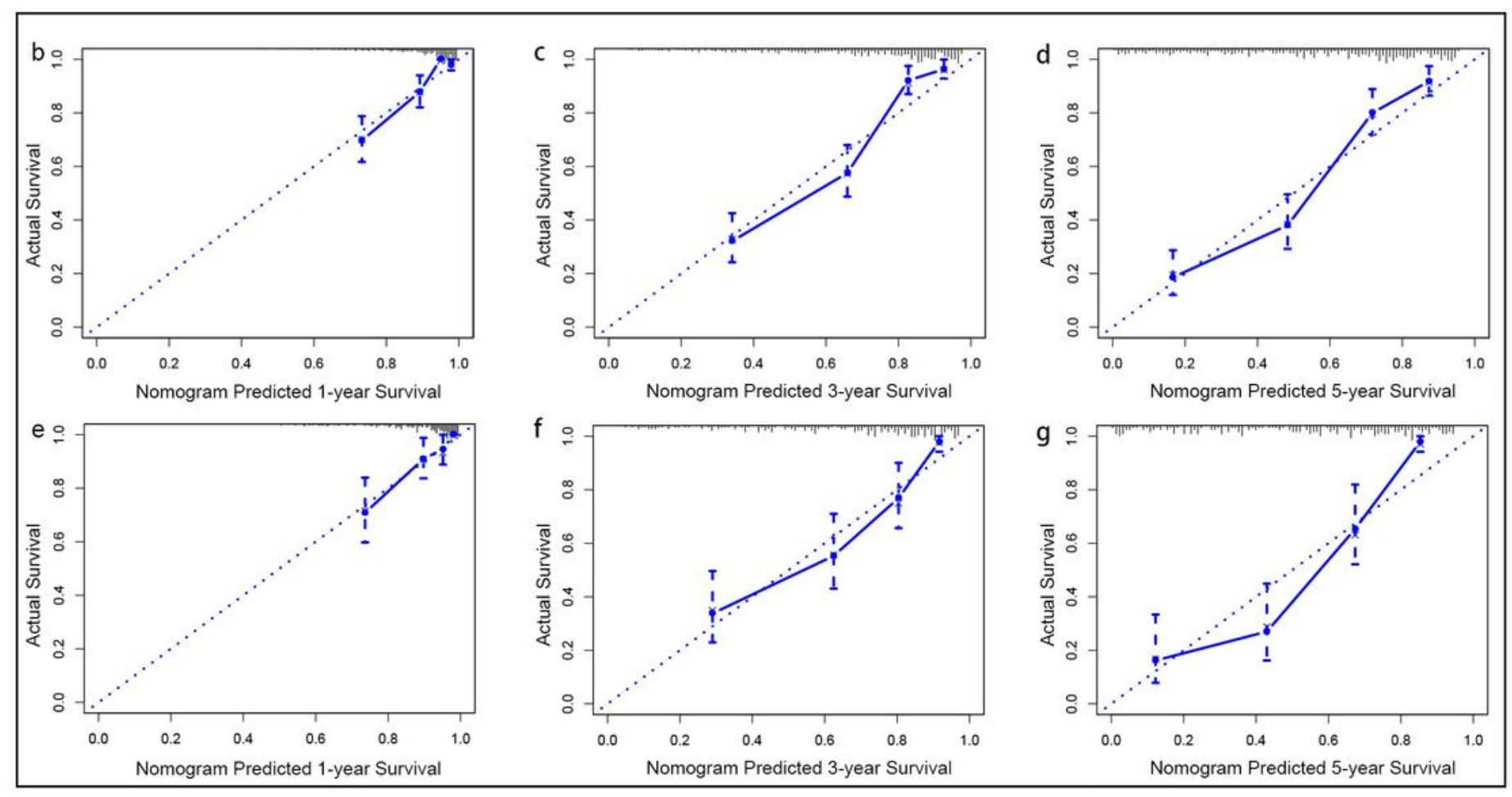

Figure 5

Nomogram for predicting the 1-, 3-, 5-year OS for LGG according to age, WHO grade, recurrent status, $1 p / 19 q$ codeletion status and risk score (a). Calibration plot for 1-, 3-, 5-year OS predicted by the nomogram in CGGA database (b-d) and TCGA database (e-g). 


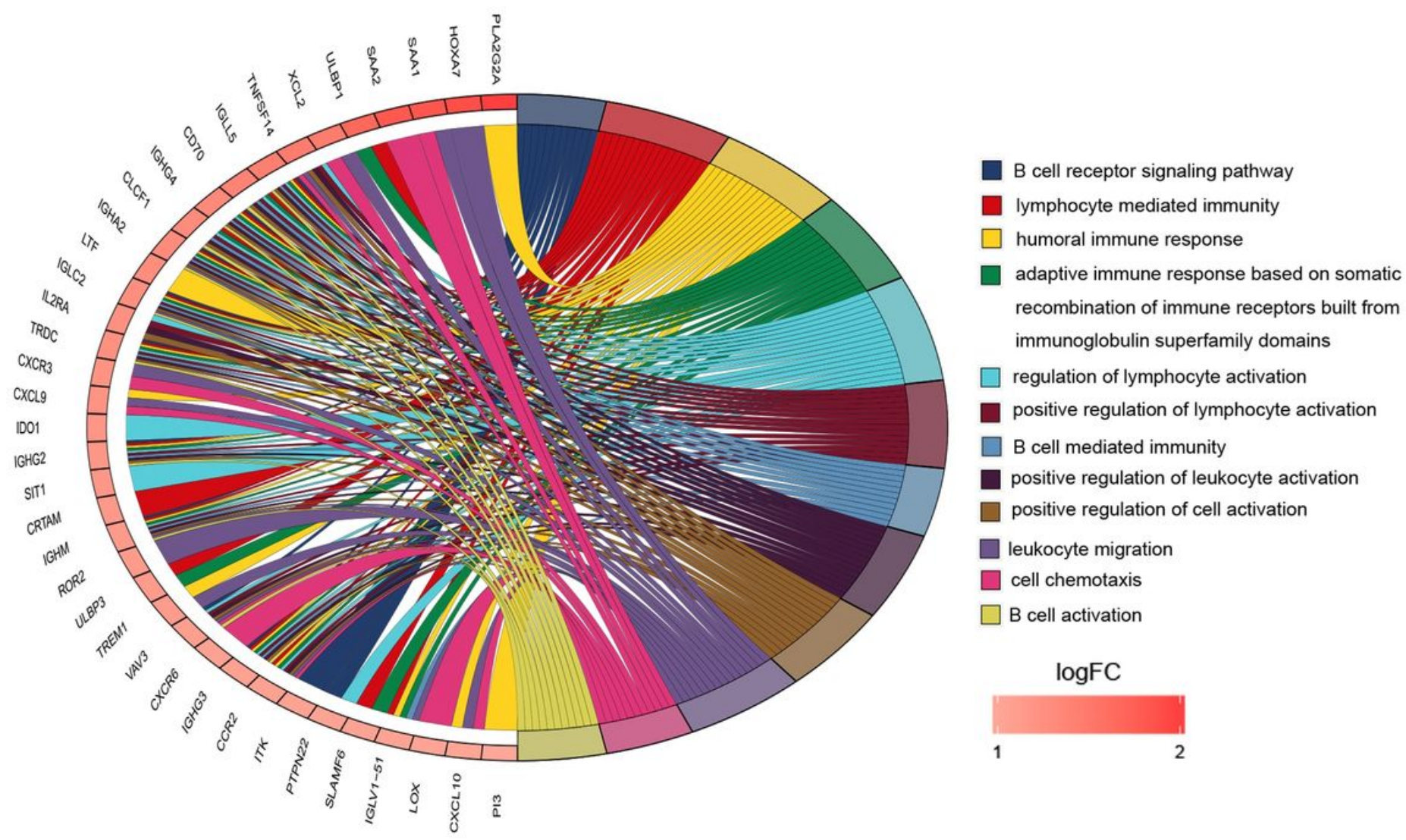

Figure 6

Chord plot showing 37 genes included in the 12 immune-related pathways. 

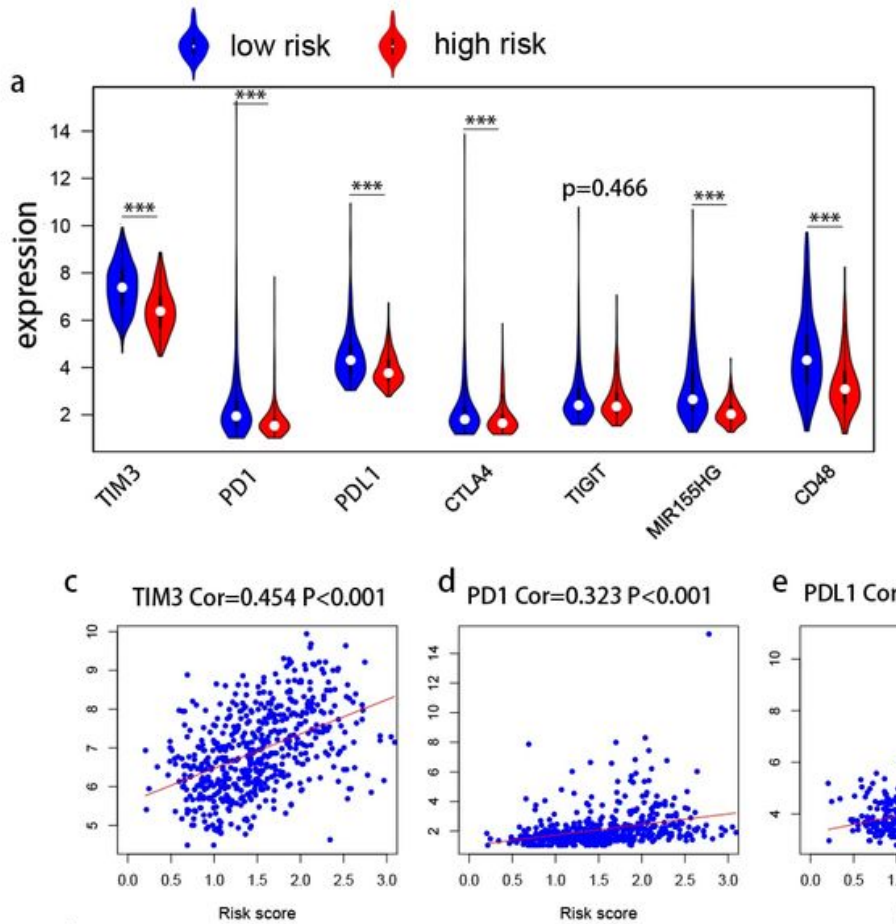

n

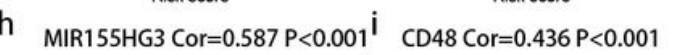

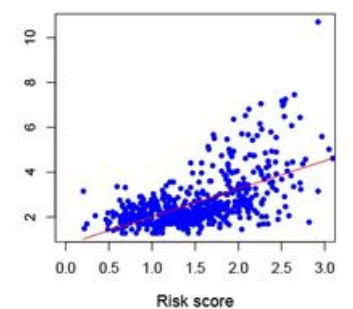

m
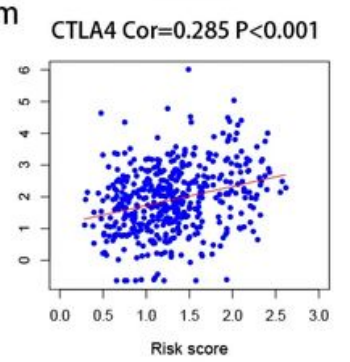

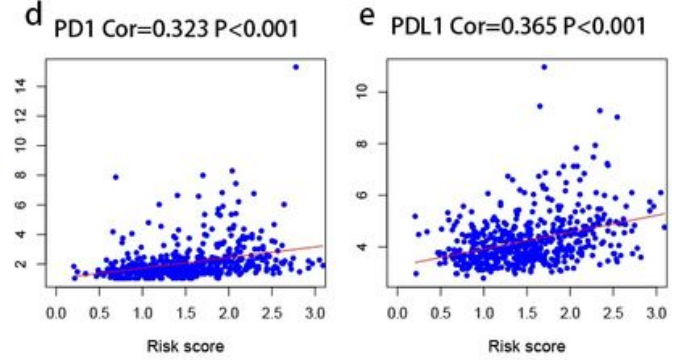

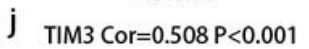

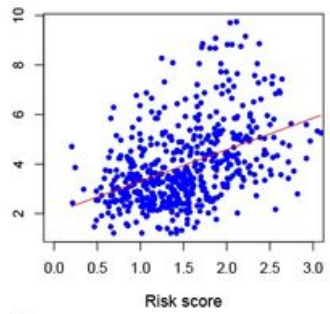

$\mathrm{n}_{\text {TIGIT Cor }=-0.121} \mathrm{P}=0.010$
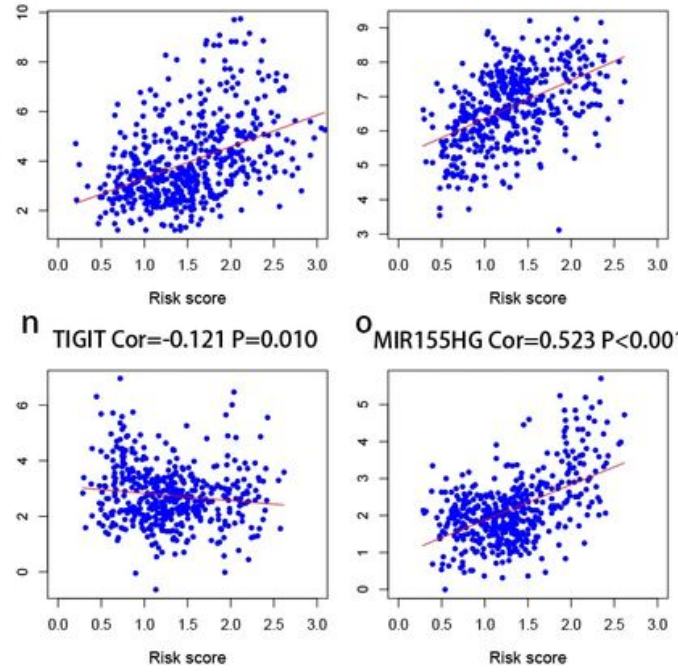
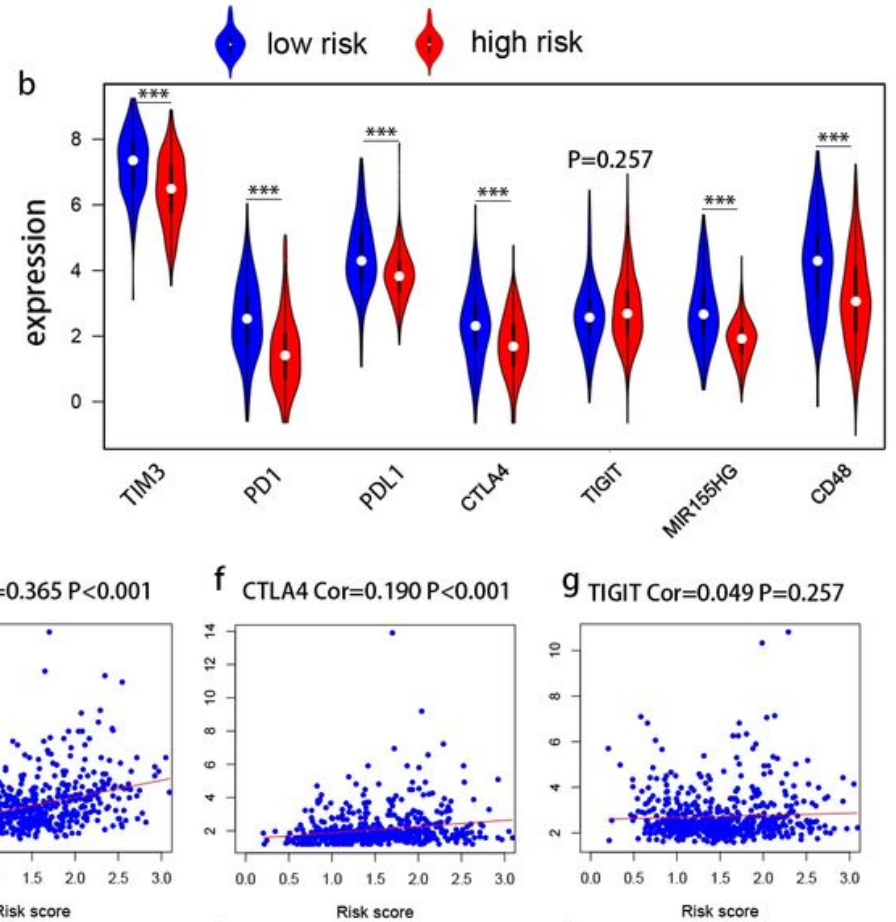

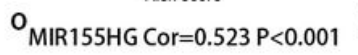
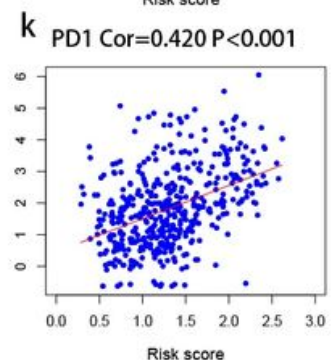

p CD48 Cor $=0.371 \mathrm{P}<0.001$

I PDL1 Cor $=0.448 \mathrm{P}<0.001$
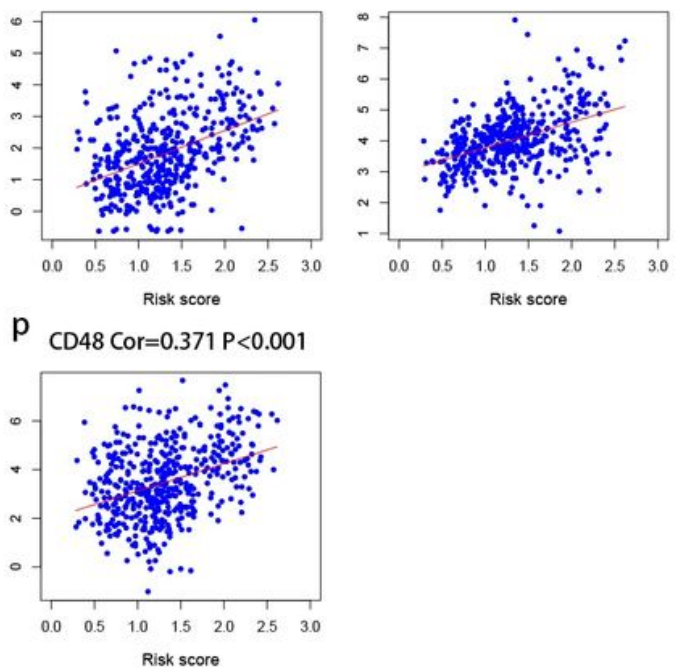

Figure 7

The expression of seven immune checkpoints (TIM3, PD1, PDL1, CTLA4, TIGIT, MIR155HG, CD48) in lowand high-risk groups in CGGA database (a) and TCGA database (b). The correlations between the immune checkpoints and risk score in CGGA database (c-i) and TCGA (j-p) database. 
a

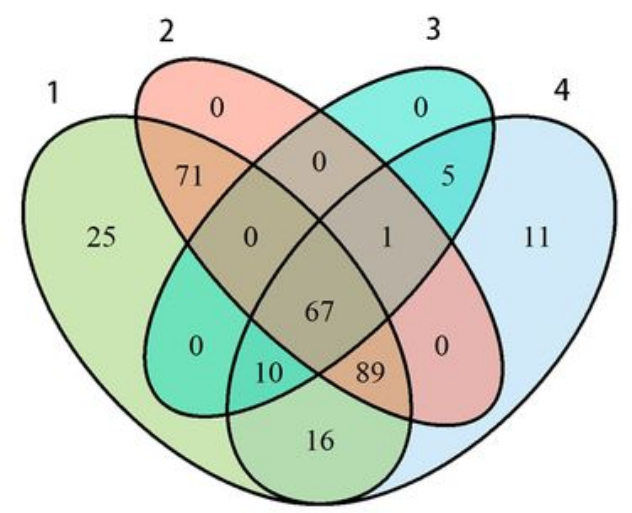

b

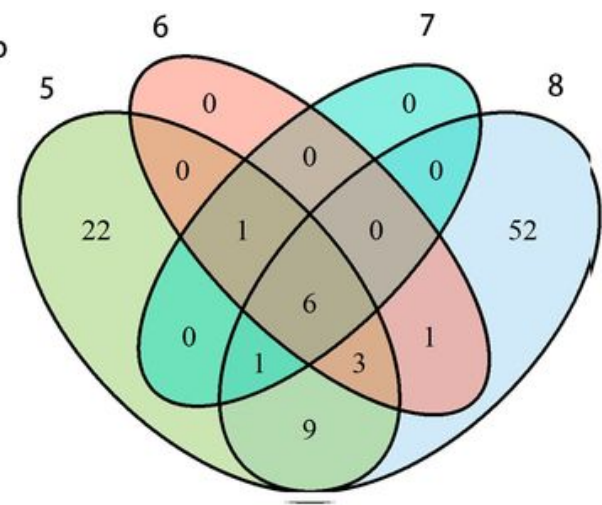

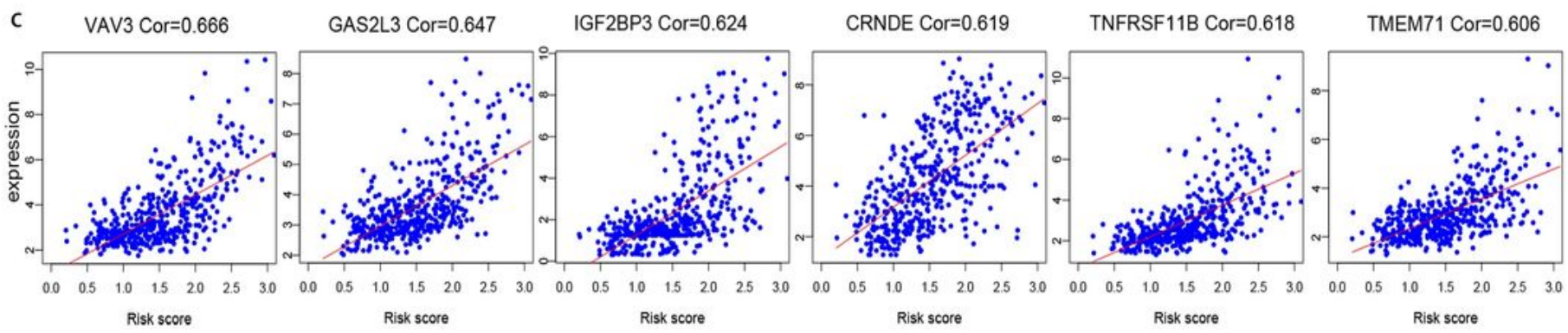

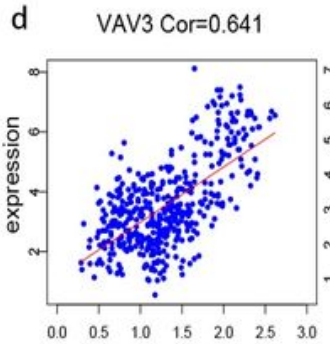

GAS2L3 Cor $=0.641$

IGF2BP3 Cor $=0.661$

CRNDE Cor $=0.622$
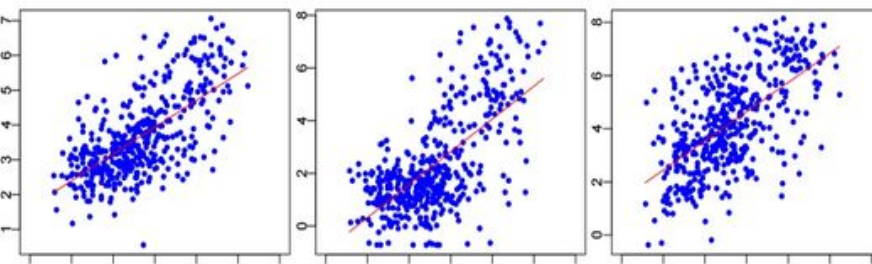

TNFRSF11B Cor $=0.641$

TMEM71 Cor $=0.696$

Risk score

Risk score

Risk score

Risk score
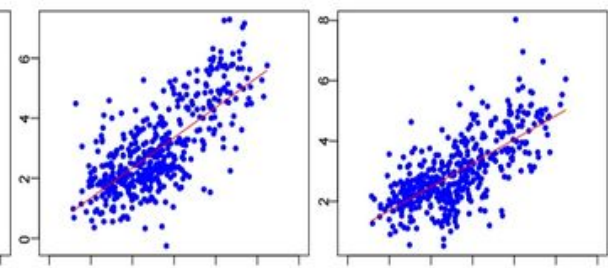

1.52

Risk score

e

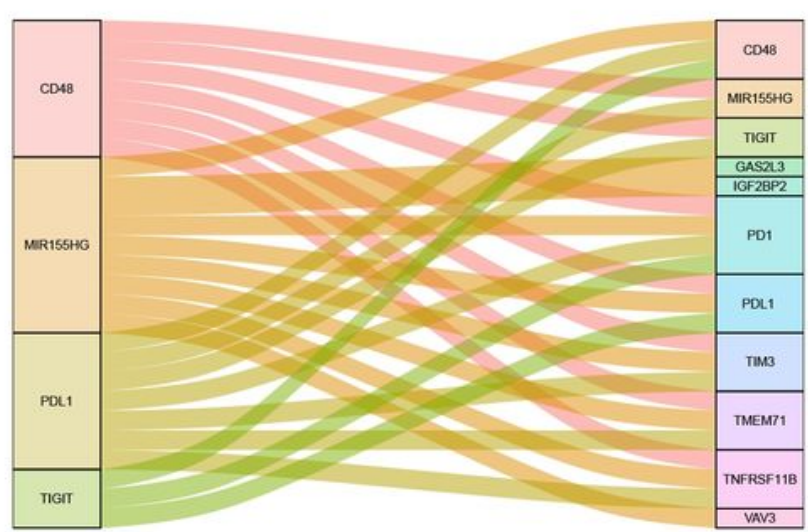

f

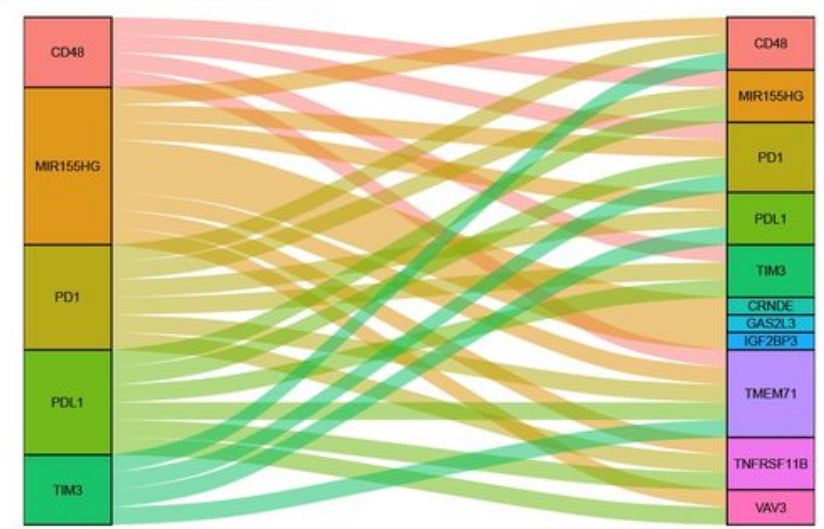

Figure 8

Venn diagrams for identification of candidate immune checkpoints. The genes are significantly associated with the OS in CGGA dataset (2) and TCGA dataset (3). The genes are independent predictors of OS in CGGA dataset (1) and TCGA dataset (4). Genes have an AUC of $>0.7(6,7)$ and a correlation value of $>0.6(5,8)$ in CGGA dataset and TCGA dataset, respectively. The correlations between six candidate immune checkpoints and the risk score in CGGA dataset (c) and TCGA dataset (d). Sankey 
diagrams showing the internal and external correlations between avowed immune checkpoints and candidate immune checkpoints in CGGA dataset (e) and TCGA dataset (f). 